\title{
Evaluation of Crystals in Formalin-Fixed, Paraffin-Embedded Tissue Sections for the Differential Diagnosis of Pseudogout, Gout, and Tumoral Calcinosis
}

Vinod Shidham, M.D., FIAC, MRCPath, Mamatha Chivukula, M.D., Zainab Basir, M.D., Ganesh Shidham, M.D.

From the Department of Pathology (VS, MC, ZB) and Division of Nephrology (GS), Medical College of Wisconsin, Milwaukee, Wisconsin

Hematoxylin-eosin (H\&E)-stained sections may not allow proper evaluation of birefringence properties of the crystals in the lesions of pseudogout, gout, and tumoral calcinosis. This study was undertaken to verify the application of a special stain that could facilitate the evaluation of the birefringence properties of these crystals for definitive diagnosis. We evaluated previously described nonaqueous alcoholic eosin staining (NAES) method based on the principle of using alcoholic eosin without hematoxylin and any other aqueous reagents for staining of formalin-fixed, paraffin-embedded tissue sections. Two observers, in a blinded fashion, evaluated the sections stained with routine $H \& E$ and NEAS method without the knowledge about clinical diagnosis. All pseudogout (nine sections from seven cases) and gout (eight sections from five cases) lesions demonstrated birefringence in the sections stained with NAES method. H\&E-stained sections showing the respective diagnostic histomorphology failed to demonstrate the birefringent crystals by polarizing microscopy in all the eight sections from gout and in seven of nine sections from pseudogout. Only two H\&E-stained sections showed scant calcium pyrophosphate dihydrate (CPPD) crystals in pseudogout. None of the three sections from two cases of tumoral calcinosis showed birefringence with either stain. We conclude that CPPD in pseudogout and monosodium urate in gout may not polarize in the routine H\&E-stained sections. How-

Copyright $($ C) 2001 by The United States and Canadian Academy of Pathology, Inc.

VOL. 14, NO. 8, P. 806, 2001 Printed in the U.S.A.

Date of acceptance: April 11, 2001.

This study was presented in part at the Fall Meeting of American Society of Clinical Pathologists/College of American Pathologists, at San Diego, CA, October 14-19, 2000 .

Address reprint requests to: Vinod B. Shidham, M.D., FIAC, MRCPath, Department of Pathology, Medical College of Wisconsin, $9200 \mathrm{~W}$. Wisconsin Avenue, Milwaukee, WI 53226; e-mail: vshidham@mcw.edu; fax: 4148058444 . ever, polarizing microscopy of sections stained with NAES method allowed demonstration of CPPD crystals with positive birefringence in pseudogout, MSU crystals with negative birefringence in gout, and calcium hydroxyapatite crystals without birefringence in tumoral calcinosis. Section stained with NAES method is a significantly useful adjunct to the routine $H \& E$ stain for proper evaluation of the crystals under polarizing microscope in these lesions.

KEY WORDS: Birefringence, Calcium hydroxyapatite, Calcium pyrophosphate dihydrate, Chondrocalcinosis, Gout, Monosodium urate, Polarizing microscope, Pseudogout, Tumoral calcinosis.

Mod Pathol 2001;14(8):806-810

Pseudogout, gout, and tumoral calcinosis may be a diagnostic challenge during the interpretation of biopsy material $(1,2)$. In pseudogout, calcium pyrophosphate dihydrate (CPPD) crystals are deposited in soft tissue, cartilage, and bone, leading in some cases to symptoms mimicking gout. Clinicians prefer the term CPPD crystal deposition disease or pyrophosphate arthritis. Deposition of CPPD crystals leading to radiologic and pathologic calcification of cartilage is called chondrocalcinosis. Like monosodium urate (MSU) crystals in gout, the CPPD crystals in pseudogout are typically seen as amorphous basophilic to amphophilic material with or without surrounding foreign-body giant cell reaction (1). Tumoral calcinosis also shows basophilic calcospherites of calcium hydroxyapatite (CHA) with surrounding foreign-body giant cell reaction (2) and may be misinterpreted occasionally as gout or pseudogout affecting the clinical management. CHA is also present in other lesions, such as soft-tissue chondroma with calcification and in posttraumatic ligamentous calcification, and may be considered in the differential diagnosis with tophus (2). Histological features are usually sugges- 
tive (1-8) but may require application of polarizing microscope to confirm the nature of crystals during the differential diagnosis for proper clinical management (9). CPPD and MSU crystals may not show birefringence in routinely stained hematoxylineosin (H\&E) sections. However, they demonstrated birefringence in the sections stained with nonaqueous alcoholic eosin staining (NAES) method (1012). The present study was undertaken to evaluate this method as an ancillary special stain with routine H\&E-stained sections for the differential diagnosis of the above lesions.

\section{MATERIALS AND METHODS}

We studied 20 tissue blocks from 14 cases. All the biopsy specimens were fixed in $10 \%$ buffered formalin for $>6$ hours but $<12$ hours and processed for paraffin embedding. They were divided into three groups based on the histological features and clinical findings:

1. Pseudogout: nine blocks, seven cases (two blocks with bone in addition to the surrounding soft tissue were decalcified before processing for paraffin embedding).

2. Gout - eight blocks, five cases, with tissue fragments $>2$-mm cores (10-12).

3. Tumoral calcinosis-three blocks, two cases. (One case in this group was initially misdiagnosed as calcification suggestive of pseudogout on routine H\&E-stained sections).

The sections were stained with H\&E, using Meyer's hematoxylin for 15 minutes without differentiation by acid alcohol. The respective next-level sections were stained with NAES method, which is based on the principle of not passing the tissue section through hematoxylin and any other aqueous reagent. This prevents masking of the CPPD and CHA crystals with hematoxylin. It also prevents the dissolution and loss of MSU crystals by aqueous reagents. Staining with nonaqueous stain visualizes the tissue architecture, allowing the examiner to appreciate the relationship of crystals with surrounding tissue. Red-colored alcoholic stain like alcoholic eosin was preferred because it matched the red background during polarizing microscopy with the compensator. We used $0.5 \%$ alcoholic eosin Y (Shandon Inc, Pittsburgh, PA) during this study. As described previously (10-12), the glass slides with 5 - to 7 - $\mu \mathrm{m}$-thick paraffin sections were progressively and sequentially dipped through the following reagents:

1. Deparaffinize the sections in xylene $(60$ seconds).

2. Xylene (six dips or 20 seconds).

3. Absolute alcohol (six dips or 20 seconds).

4. Absolute alcohol (six dips or 20 seconds).
5. $0.5 \%$ alcoholic eosin Y (Shandon Inc; six dips or 20 seconds).

6 . Wash the excess of $0.5 \%$ alcoholic eosin Y with absolute alcohol (six dips or 20 seconds).

7. Absolute alcohol (six dips or 20 seconds).

8. Absolute alcohol (six dips or 20 seconds).

9. Xylene (six dips or 20 seconds).

10. Xylene (six dips or 20 seconds).

11. Coverslip with nonaqueous mounting medium, such as S/P brand ACCU. MOUNT 60 (Baxter Healthcare Corporation, McGaw Park, IL).

The slides stained with H\&E (control) and NAES (study) method were examined by light microscope for the histological features. They were also examined with polarizing microscope for the evaluation of crystal birefringence (11, 13-15). Two observers without the knowledge of the clinical diagnosis evaluated these features.

\section{RESULTS}

In Group A (pseudogout), all nine slides stained with NAES method showed numerous rhomboidto rod-shaped pleomorphic crystals with blunt ends under light microscope (Table 1, Fig. 1A4). They demonstrated birefringence under polarizing microscope (Fig. 1A4). These crystals were blue when the long axes of the crystals were parallel to the axis of the slow ray of the first-order red compensator and were yellow when the long axes of the crystals were perpendicular to the axis of the slow ray under polarizing microscope. When the axis of the slow ray was rotated, the crystals changed the colors concordant with the pattern of positive birefringence, consistent with CPPD crystals. All H\&Estained sections showed histological features consistent with pseudogout (Fig. 1A1). When these H\&E-stained sections were examined under polarizing microscope, only two sections showed scant crystals with weak positive birefringence (Table 1), and the remaining seven sections did not demonstrate any birefringence (Fig. 1A2). Two blocks from the decalcified tissue belonged to the latter group of seven sections.

All the eight slides from Group B (gout) stained with NAES method demonstrated MSU crystals by light microscope. The crystals were yellow-brown aggregates of the needle-shaped structures simulating stacks of hay (Table 1, Fig. 1B3). They demonstrated diagnostic birefringence under the polarizing microscope (Fig. 1B4). The crystals demonstrated yellow color when their long axes were parallel to the axis of the slow ray, and blue color when their long axes were perpendicular to the axis of the slow ray of the firstorder red compensator. The rotation of the slow-ray axis changed the colors of the crystals corresponding to the pattern of negative birefringence, consistent 
with MSU crystals. They did not demonstrate any birefringence in the sections stained with H\&E (Fig. 1B2); however, the histologic features consistent with gouty tophus were observed in all H\&E-stained sections (Table 1, Fig. 1B1).

In Group C (tumoral calcinosis), all three sections stained with H\&E showed amorphous basophilic psammoma body-like calcospherites with surrounding foreign-body giant cell reaction (Fig. C1). None of the sections stained with either H\&E or NAES method showed any birefringence, which was consistent with CHA crystals (Table 1, Fig. 1, C2, C4). Both the observers concurred with the interpretations in all cases with similar results.

\section{DISCUSSION}

CPPD crystals in pseudogout, MSU crystals in gout, and CHA crystals in the lesions such as tumoral calcinosis, soft tissue chondroma with calcification, and posttraumatic ligamentous calcification have characteristic features that can be helpful during the biopsy interpretation. MSU crystals dissolve in water and are lost from H\&E-stained sections $(11,16)$. Depending on the type of hematox- ylin used, CPPD crystals may be masked by the hematoxylin and lose their birefringence. Because of this, pseudogout associated with mass lesion may be misinterpreted as tumoral calcinosis and vice versa $(1,17)$. As observed in gout, different types of crystals may coexist in the same lesion (6), confusing the issue further if the crystals cannot be evaluated by polarizing microscopy. These crystals have different morphological and birefringence patterns. Both MSU and CPPD crystals are birefringent, whereas CHA crystals do not demonstrate any birefringence under polarizing microscope. The theory of polarizing microscopy has been described previously in detail $(14,15)$.

In Group A (pseudogout), only two of nine H\&E sections demonstrated scant CPPD crystals with weak birefringence under polarizing microscope; however, all nine sections stained with NAES method demonstrated numerous pleomorphic-, rod-, and rhomboid-shaped CPPD crystals with diagnostic positive birefringence under polarizing microscope. In routinely stained H\&E sections, the birefringence of CPPD crystals may be completely lost or markedly reduced because of the masking effect of hematoxylin. This may be due to the affin-

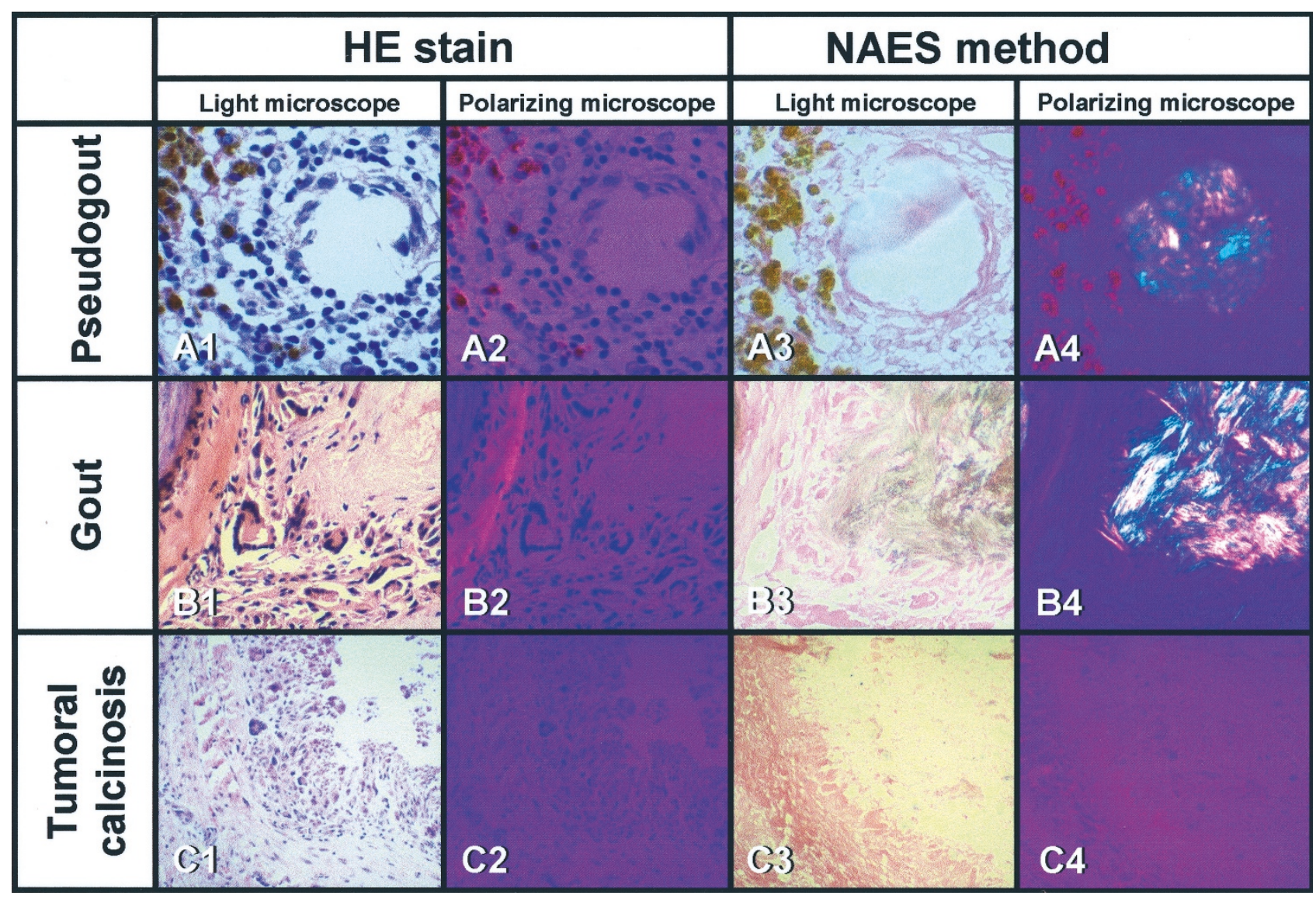

FIGURE 1. Findings under a light microscope and a polarizing microscope (A1, A2, A3, A4, B1, B2, B3, B4, 250×; C1, C2, C3, 100×). Sections stained with $H \& E$ demonstrated relevant histopathology (A1, B1, C1); however, they did not show any birefringent crystals under a polarizing microscope (A2, B2, C2). Sections stained with NAES method demonstrated birefringent crystals under polarized light in pseudogout (A4) and gout (B4) but did not show any birefringent crystals in tumoral calcinosis (C4). 


\begin{tabular}{|c|c|c|c|c|c|c|c|}
\hline \multirow{2}{*}{ Group } & \multirow{2}{*}{ Diagnosis } & \multirow{2}{*}{$\begin{array}{l}\text { Patients } \\
\text { (No.) }\end{array}$} & \multirow{2}{*}{$\begin{array}{l}\text { Tissue Blocks } \\
\text { (No.) }\end{array}$} & \multicolumn{2}{|c|}{$\begin{array}{l}\text { Birefringence Under } \\
\text { Polarizing Microscope }\end{array}$} & \multicolumn{2}{|c|}{ Findings under Light Microscope } \\
\hline & & & & $\begin{array}{l}\text { H\&E stain } \\
n(\%)\end{array}$ & $\begin{array}{c}\text { NAES method } \\
n(\%)\end{array}$ & H\&E stain & NAES method \\
\hline A & Pseudogout & 7 & 9 & $2^{a}(22)$ & $\begin{array}{l}9 \text { (100) } \\
\text { positive } \\
\text { birefringence }\end{array}$ & $\begin{array}{l}\text { amorphous, pale, basophilic } \\
\text { to amphophilic material } \\
\text { with or without foreign } \\
\text { body giant cell reaction }\end{array}$ & $\begin{array}{l}\text { pale, translucent, coarse, } \\
\text { rod- to rhomboid-shaped } \\
\text { crystals with blunt ends }\end{array}$ \\
\hline B & Gout & 5 & 8 & None & $\begin{array}{l}8 \text { (100) } \\
\text { negative } \\
\text { birefringence }\end{array}$ & $\begin{array}{l}\text { feathery, amphophilic } \\
\text { material with foreign body } \\
\text { giant cell reaction }\end{array}$ & $\begin{array}{l}\text { yellow-brown, needle-shaped } \\
\text { crystals with pointed ends } \\
\text { distributed singly or in } \\
\text { groups simulating hay } \\
\text { stacks }\end{array}$ \\
\hline $\mathrm{C}$ & $\begin{array}{l}\text { Tumoral } \\
\text { calcinosis }\end{array}$ & 2 & 3 & None & $\begin{array}{l}0 \text { no } \\
\text { birefringence }\end{array}$ & $\begin{array}{l}\text { basophilic amorphous, } \\
\text { globular structures with } \\
\text { surrounding giant cell } \\
\text { reaction }\end{array}$ & $\begin{array}{l}\text { pale, translucent, coarse, } \\
\text { globular calcospherites }\end{array}$ \\
\hline
\end{tabular}

${ }^{a}$ Scant occasional birefringent crystals difficult to interpret were present. These sections were not from the decalcified blocks. H\&E, hematoxylin \& eosin; NAES, nonaqueous alcoholic eosin staining.

ity of hematoxylin for calcium in these crystals that may still transmit the light but does not polarize it (Fig. 1A2). One of our cases of tumoral calcinosis was misinterpreted initially based on H\&E stain as suggestive of pseudogout. However, with the NAES method, globular CHA crystals were clearly differentiated because of lack of birefringence from the positively birefringent CPPD crystals of pseudogout and negatively birefringent MSU crystals in gout (Fig. 1).

Staining with Alizarin red for the calcium component of CPPD and von Kossa method for the phosphate component of CPPD have been suggested for staining these crystals (4). Previously described techniques like deGalantha stain and Gomori methenamine silver method for MSU are also not specific and do not offer additional advantage over the routine $\mathrm{H} \& \mathrm{E}$ sections $(7,9,18)$. In Group B (gout), all the sections stained with NAES method demonstrated needle-shaped, negatively birefringent crystals with pointed ends, consistent with MSU; however, none of these sections stained with $\mathrm{H} \& \mathrm{E}$ demonstrated the birefringence of MSU crystals (Table 1, Fig. 1). The NAES method can demonstrate these crystals under polarized light with good inter-observer reproducibility. The sections stained with it can highlight the characteristic morphology with diagnostic birefringence and is a significantly useful ancillary special stain during the interpretation of H\&E-stained sections of these lesions.

The interpretation of CPPD crystals in the sections stained with NAES method was not interfered with by the decalcification process of the tissue. Additional study specifically evaluating the effects of decalcification on the results with NAES method in a larger series may be indicated. However, on the basis of our two cases in this study, decalcification does not appear to interfere with the birefringence of CPPD crystals. Lack of interference effect of decalcification on MSU crystals has been described previously $(10,11)$. Tumoral calcinosis, soft tissue chondroma with calcification, and posttraumatic ligamentous calcification may not be of concern with reference to birefringence, as CHA crystals present in these lesions are not birefringent. CHA crystals may also be associated with other conditions like trauma, scleroderma, renal failure, hyperparathyroidism, sarcoidosis, metastatic disease, myeloma, or hypercalcemic/hyperphosphatemic states $(1,2)$.

In this study, formalin-fixed, paraffin-embedded tissue sections were used. However, as discussed previously, if scant MSU crystals are anticipated, the tissue may be fixed preferably in alcohol to avoid any loss of scant crystals in aqueous fixatives like formalin. For practical purposes, tissue $>2 \mathrm{~mm}$ core size and processed within 12 hours of fixation in formalin should retain significant amounts of MSU crystals that can be demonstrated by the NAES method $(11,12)$. As CPPD and CHA crystals are not water soluble, formalin fixation does not interfere with their interpretation. Tissue sections thicker than $7 \mu \mathrm{m}$ may be used if the quantity of material to be studied in tissue is anticipated to be scant $(11,12)$.

In summary, the NAES staining method prevented the dissolution of MSU crystals from tissue sections and preserved the birefringence property of CPPD crystals for proper evaluation under polarizing microscope and light microscope. Depending on the type of method used for H\&E staining, the birefringence of CPPD crystals may be masked by hematoxylin. We used $0.5 \%$ alcoholic eosin Y without passing through any aqueous reagent including hematoxylin. This prevented the masking interference by hematoxylin during the evaluation of birefringence of CPPD crystals. NAES method is an 
useful adjunct to the routine H\&E stain for demonstrating the diagnostic positive birefringence of rhomboid- to rod-shaped pleomorphic CPPD crystals in pseudogout, the needle-shaped MSU crystals with negative birefringence in gout, and the psammoma body-like calcospherites of CHA crystals without birefringence in tumoral calcinosis.

Acknowledgments: We thank Christopher Chartrand, HT (ASCP) for histochemistry assistance and Sushrut Shidham for the expert secretarial assistance. We also appreciate the expert histology assistance of histotechs from the Department of Pathology, Clement J. Zablocki Veterans Affairs Medical Center, Milwaukee, WI. We thank Hongyung Choi, M.D. from Clement J. Zablocki Veterans Affairs Medical Center, Milwaukee, WI and Gordon E. Lang, M.D. and Ellen Manlucu, M.D. from St. Mary's Hospital, Milwaukee, WI for contributing the cases to this study. We are grateful to Carl Becker, M.D. and Richard Komorowski, M.D. at the Department of Pathology, Medical College of Wisconsin, Milwaukee, WI, for reviewing this manuscript and providing valuable comments.

\section{REFERENCES}

1. Bullough PG. Joint diseases. In: Sternberg SS, Antonioli DA, Mills SE, Carter D, Oberman HA, editors. Diagnostic surgical pathology. 3rd ed. Philadelphia: Lippincott, Williams \& Wilkins; 1999. p. 223-61.

2. Enzinger FM, Weiss SW, editors. Soft tissue tumors. 3rd ed. St. Louis, MO: Mosby; 1995.

3. Ackerman AB, Chongchitnant N, Sanchez J. Inflammatory diseases. In: Histological diagnosis of inflammatory skin diseases, an algorithmic method by pattern analysis. 2nd ed. Philadelphia: Lippincott, Williams \& Wilkins; 1997. p. 388.

4. Bullough PG. Non-neoplastic diseases of bones and joints. In: Silverberg SG, DeLellis RA, Frable WJ, editors. Principles and practice of surgical pathology and cytopathology. 3rd ed. New York: Churchill Livingstone; 1997. p. 846-7.

5. Golitz LE. Inflammatory diseases. In: Silverberg SG, DeLellis RA, Frable WJ, editors. Principles and practice of surgical pathology and cytopathology. 3rd ed. New York: Churchill Livingstone; 1997. p. 367-69.

6. Hough AJ Jr. Joints. In: Damjanov I, Linder J, editors. Anderson's pathology. 10th ed. St. Louis, MO: Mosby; 1996. p. 2637-9.

7. Rosai J. Bone and joints. In: Ackerman's surgical pathology. 8th ed. St. Louis, MO: Mosby; 1996. p. 1996-7.

8. Yeldani AA, Kaufman DG, Reddy JK. Cell injury and cellular adaptations. In: Damjanov I, Linder J, editors. Anderson's pathology. 10th ed. St. Louis, MO: Mosby; 1996. p. 368-9.

9. deGalantha E. Technique for preservation and microscopic demonstration of nodules in gout. Am J Clin Pathol 1935;5: 165-6.

10. Shidham VB, Galindo L, Gupta D, Jhala N, Shidham GB. Visualization of urate crystals in paraffin sections [abstract]. Am J Clin Pathol 1997;107:481.

11. Shidham VB, Galindo LM, Gupta D, Jhala N, Shidham GB. Urate crystals in tissue. A novel staining method for formalin-fixed, paraffin-embedded sections. Lab Med 1998; 29:109-13.

12. Shidham V, Shidham G. Staining method to demonstrate urate crystals in formalin-fixed, paraffin-embedded tissue sections. Arch Pathol Lab Med 2000;124:774-6.

13. Fiechtner JJ, Simkin PA. Urate spherulites in gouty synovia. JAMA 1981;245:1533-6.

14. Judkins SW, Cornbleet PJ. Synovial fluid crystal analysis. Lab Med 1997;28:774-9.

15. Schumacher HR Jr, Reginato AJ. Atlas of synovial fluid analysis and crystal identification. Philadelphia: Lea \& Febiger; 1991.

16. Simkin PA, Bassett JE, Lee QP. Not water, but formalin, dissolves urate crystals in tophaceous tissue samples. J Rheumatol 1994;21:2320-1.

17. Sissons HA, Steiner GC, Bonar F, May M, Rosenberg ZS, Samuels $\mathrm{H}$, et al. Tumoral calcium pyrophosphate deposition disease. Skeletal Radiol 1989;18:79-87.

18. Bancroft JD, Stevens A. Pigments and minerals. In: Bancroft JD, Stevens A, editors. Theory and practice of histological techniques. 3rd ed. New York: Churchill Livingstone; 1990. p. 262. 